\title{
The Onset of Mackey-Glass Leukemia at the Edge of Chaos
}

\author{
M. Argollo de Menezes and R.M. Zorzenon dos Santos \\ Instituto de Física, Universidade Federal Fluminense, \\ Av. Litorânea s/n, 24210-340, Niterói, RJ, Brasil \\ e-mail:marcio@if.uff.br; zorzenon@if.uff.br
}

November 28, 2018

\begin{abstract}
In this paper we revisit the Mackey-Glass model for blood-forming process, which was proposed to describe the spontaneous fluctuations of the blood cell counts in normal individuals and the first stage of chronic myelocytic (or granylocytic) leukemia (CML). We obtain the bifurcation diagram as a function of the time delay parameter and show that the onset of leukemia is related to instabilities associated to the presence of periodic windows in the midst of a chaotic regime. We also introduce a very simple modification in the death rate parameter in order to simulate the accumulation of cells and the progressive increase of the minima counts experimentally observed in the final stage of the disease in CML patients. The bifurcation diagram as a function of the death rate parameter is also obtained and we discuss the effects of treatments like leukapheresis.
\end{abstract}

\section{Introduction}

Many years ago Mackey and Glass [1] proposed two different simple models to describe chronic and acute diseases, generated by the failure of physiological control systems, which may be identified by the altered periodicity of some observable. Recently Landa and Rosemblum [2] revisited one of the models proposed to describe the respiration control and introduced a modification in the original equation in order to take into account the activity of the brain on respiration. In this paper we revisit the model proposed to describe the fluctuations in the blood cell counts in normal individuals and the initial phase of chronic myelocytic leukemia (CML).

The human blood contains a remarkable variety of cells, each type precisely designed to its own vital function. All different types of blood cells are produced in the bone marrow and all of them are originated from earlier non-functional 
cells called stem cells. The stem cells replicate repeatedly and differentiate into various kind of secondary cells. The growth and development of cells in the bone marrow are carefully controlled to produce the correct number of each type of cell necessary to keep the body healthy. The blood-forming process (hematopoiesis) is subjected to active control by mechanisms acting to buffer the system against disturbances and to provide adaptive adjustment to nonstationary conditions [3]. The hematopoiesis is regulated by feedback control systems and as other time-delayed processes [4, 通] it exhibits oscillatory and cyclic behaviors.

Most of the models proposed to describe physiological control systems consider the differentiation steps of the secondary cells as different compartments described by differential equations. In general those models have a high-dimensional parameter space ([6]- [8]) which makes it quite difficult to compare theoretical results to the experimental ones. In contrast to those more realistic, but rather complicated models, Mackey and Glass [1] proposed a very simple model based on a single time-delayed differential equation, with parameters estimated from experimental data. The change of the time delay parameter leads to different kind of oscillations of the cell counts which may be associated to healthy and non-healthy behaviors when compared to experimental data obtained by Gatti et al [9]. The onset of an abnormal dynamics, which may be associated to the onset of leukemia is then obtained in this model by the gradual tuning of a control (time-delay) parameter. Since its proposal this model has been mostly used as a paradigm for non-linear systems ([10]- [14]), and less attention was paid to its biological applications.

In this paper, we show that this simple model that generates very complex dynamical behaviors for cell counts, is more robust than thought to reproduce the onset of leukemia and it is also possible to use it to reproduce the two phases of leukemia and the effects of some treatments. The observed instabilities that occur at the onset of leukemia can be obtained for different values of the time delay parameter, corresponding to different possibilities of the system to achieve the edge of chaos. In other words, the instabilities resulting from complex interactions involved in the physiological control systems that may describe the oscillations observed in CML patients correspond to an state hovering between the incoherence of chaos and the spontaneous order found in periodic behaviors (15).

In section 2 we present the biological motivation that inspired the model discussed here and other experimental data concerning the discussions we present along the paper. In section 3 we introduce the model. In section 4 we present the results obtained. We first obtain the bifurcation diagram in function of the time delay parameter for the Mackey-Glass model and show that the onset of leukemia is related to instabilities associated to transition regions between chaotic and periodic regimes, usually refereed in the literature as the edge of chaos. These transition regions occur for a set of different values of the timedelay parameter and not only for a unique value of this parameter. The bifur- 
cation sequence observed in this diagram is quite uncommon and presents only some of the periods suggested by the authors [1, 5]. We also introduce a simple modification in the death rate parameter of this model in order to simulate the progressive increase of the minima counts in phase II of CML. The bifurcation diagram in function of the death rate parameter is also obtained and the effects of leukapheresis treatments is also discussed. Our conclusions are summarized in section 5 .

\section{The Biological Motivation}

It has been observed spontaneous fluctuations with periodic behavior in the peripheral blood cell counts of healthy individuals, in chronic myelocytic leukemia (CML) patients [16, 17] as well as in other pathological states [5]. In healthy individuals constant or mild oscillations of the peripheral-blood leukocyte-counts occur with periods varying from 14 to 24 days [16], while in many patients with CML these oscillations have periods varying from 30 to 120 days (16, 18]), with a cell accumulation process observed in the final stage (phase II) of the disease. Morley et al [16] suggested that in patients with cyclic leukocytosis and CML, the bone marrow is either unduly sensitive to feedback stimulus or being excited intermittently by an abnormal stimulus, generated outside the marrow.

From long term observations Gatti et al. [9] showed that the onset of leukemia $(\mathrm{CML})$ is characterized by two distinct phases: phase I, a stable 15month period, from time of diagnosis, during which leukocyte counts peaked every 72 days and then returned to the baselines level; and phase II, a 18month period during which the minima levels of leukocyte counts became progressively higher indicating that those cells were accumulating in the peripheral blood. According to them, the increased marrow production in the disease does not represent a life-threatening situation, until cell accumulation begins. During phase I, the leukocyte counts return to baseline levels, indicating that the overproduction of cells in the peak of a given cycle could be cleared from the peripheral blood before the beginning of the next cycle. In contrast, in phase II, the leukocytes accumulate in the peripheral blood of the patient, suggesting primarily an increase in the cell production or a progressive loss of cell-clearing capacity with little or no changes in the production capacity [9].

Patients with CML were submitted to leukapheresis treatments in order to test out if this kind of treatment would be able to reverse the rising leukocyte levels providing a good therapy to maintain the patient healthy for years. The leukapheresis consists of 8 weeks of intense treatment to remove about $30-50 \%$ of the mature and premature leukocytes from marrow and peripheral blood. According to the results obtained [9], those treatments are able to retrieve the system from the behavior of phase II to the behavior observed in phase I, with low amplitude cycles and minima counts. However, after one year, subsequent cycles (of 72 days each) indicated re-accumulation of cells in the peripheral 
blood. In other words, these studies clearly demonstrated that leukapheresis is effective for the rapid reduction of elevated cell counts, but long-term treatment has not improved survival of the patients with CML, since this kind of treatment do not alter the mechanisms underlying the disease process.

Nowadays this kind of treatment is not used anymore, and have been replaced by other treatments like chemotherapy and radiotherapy that in some cases may lead to the patient cure. The mechanisms underlying the effectiveness (or not) of those modern treatments are not clear-they act by producing an intense immunosupression followed by an intense activation of the bone marrow in order to recover the system, involving different kinds of drugs- and it would be impossible to discuss its effects on this very simple model. However, it is possible to analyze and simulate the effects of leukapheresis in the MG model as will be shown in section 4 .

\section{The Model}

The Mackey-Glass model [1] relates the number of precursor and mature cells resident on the bone marrow to the number of cells released into the blood stream. The time evolution of an homogeneous population of mature, circulating cells of density $P$ is then given by:

$$
\frac{d P(t)}{d t}=\frac{\beta_{0} \theta^{n} P_{\tau}}{\theta^{n}+P_{\tau}^{n}}-\gamma P(t)
$$

where the first term in the right hand side corresponds to the cellular production, which is described by a (Hill) function of $P_{\tau}=P(t-\tau)$, where $\tau$ represents the delay between the production of the precursor cells and the release of the mature cells into the blood. The average (density independent) death rate of the population is given by $\gamma$. The constant values of $\beta_{0}, \theta, n$ and $\gamma$ are estimated from experimental data [1].

Like other theoretical models $([\overline{6}]-[\beta])$, the Mackey-Glass model associates the onset of cyclic leukocytosis and leukemia with instabilities in the dynamics of the model. They found that for $\tau=6$ days the solution has low amplitude oscillations with period of 20 days, which describes qualitatively the mild oscillations observed in the circulating levels of leukocytes in normal healthy adults [16]. For $\tau=20$, the numerical solution reproduces the altered periodicity observed in phase I of the diagram of circulating leukocytes as a function of time of patients with diagnosed CML [9].

\section{$4 \quad$ Results}

By standard fourth-order Runge-Kutta integration techniques the numerical solution for the equation is obtained, where we adopt the same set of parameters 
of the original model [1]. In order to characterize the dynamical instabilities associated to the onset of leukemia we have obtained the bifurcation diagram of the local maxima of the time evolution of $P(t)$ as a function of the time delay parameter $\tau$ as shown in figure 1. This diagram was built from the analysis of the cross section of the return map for different values of $\tau$. By increasing the time-delay parameter the oscillations become unstable and new cycles with different periods appear, leading to aperiodic and then to chaotic behavior. In contrast with the findings of Mackey-Glass, we found a quite uncommon bifurcation sequence: $1,2,3,6,7,14$, etc. with windows of period 6 and 9 in the midst of the aperiodic regime. We also note the appearance of a branch of sparse points just above $\tau=17$. This branch persists when we vary the initial conditions.

We have observed that the very same altered periodicity observed for $\tau=20$ (which was associated to the onset of the cyclic leukocytosis by Mackey and Glass [1]) could also be reproduced for other values of $\tau$, for instance, $\tau=18.6$ and 21.2. The common feature among these values of the time delay parameter is that they correspond to the edge of the periodic windows which appear in the midst of aperiodic regime. This indicates the robustness of the model to describe the disturbances that would correspond to the onset of leukemia.

In this model, the regular behavior of the blood-forming process and the pathological states of disease correspond to different attractors of the dynamics. The standard methods of characterization of the attractors can be applied for small values of $\tau$ (periodic behavior), whose attractors have low embedding dimension, and for large values of this parameter (chaotic behavior), whose embedding dimension of the attractors can be approximated to infinity, for which simple asymptotic solutions exist 110, 11. Nevertheless, for intermediate values of this parameter the attractors are embedded in a high-dimensional space and the characterization becomes quite difficult, specially in cases like this one, which involves time-delayed information. As far as we know up to now the available techniques do not allow a precise characterization of the dynamics for intermediate values of $\tau$. In figure 2 we show the power spectra for four values of $\tau$ corresponding to the three different dynamical behaviors described above. Our results indicate that the special values corresponding to the onset of leukemia would correspond to a quasi-periodic behavior: the power spectrum has numerous peaks and presents a slow decay to zero. This behavior should correspond to the zero value of the Lyapunov exponent. Nevertheless, because of the difficulties mentioned above, the calculations performed are still not conclusive.

As mentioned before, the phase II of the common pattern observed in CML patients may be associated to either a failure of the cell-clearing capacity of the system or a over-production of immature cells (the cells lose the ability to mature and differentiate) which leads to the accumulation of cells and to a progressive increase of the minima counts in the leukocyte population [9]. In order to simulate the phase II, we introduce a simple modification to the death rate 
that would correspond to a failure in the cell-clearing capacity. Modifications in the cell production, in order to simulate the loss of the cell's ability to mature and differentiate is not straightforward and will be discussed elsewhere. The different dynamical behaviors observed in the two distinct phases, will be related to changes in the death rate, since in phase II it will be no longer constant. The death rate $\gamma$ will be described during phase II by a step function that diminishes by $10^{-4}$ every 60 days. This simple modification simulates a system that slowly loses the ability to remove cells, and reproduces qualitatively the increase of the minima counts, observed by Gatti et al [9] in phase II of the disease, as shown in figure 3. We have also tried other possible functions, but this one was more appropriate to describe the accumulation of cells observed in phase II.

Figure 3 is obtained for the same set of parameters used in the original model. We have arbitrarily chosen to let the system evolve with $\gamma$ constant until $t=4900$ (which would correspond to the end of phase I) and after this point the death rate begins to decrease. The slope of the linear growth of the minima counts in our simulations has the same order of magnitude as the equivalent slope in the experimental data.

In figure 1 we present the bifurcation diagram as a function of $\gamma$, for $\tau=20$, in the range of interest. The diagram shows a bifurcation sequence which ends up in a limited region from 0.08 to 0.12 of aperiodic behavior, above which the system becomes periodic again. In the midst of the aperiodic region we also find windows of periodicity 6 .

We also simulate the effects of leukapheresis treatments during the phase II. We let the system evolve during some time (600 days) in phase II and then we simulate the first leukapheresis, in order to test if this cleaning procedure would revert the raising of the minima counts. The treatment is simulated maintaining the density of cells constant during 60 days (equivalent to the 8 weeks of real treatment) in a level that corresponds to $60 \%$ of the value of the density in the beginning of the protocol [19]. For $\tau=20$, after 60 days the system does not have memory of what happened before the treatment. After each treatment we let the system evolve under the same conditions, since the treatment only removes the excess of cells accumulated and do not interferes on the underlying mechanisms that could generate the disturbance. Just after the treatment the minima counts returned to the baseline levels, but after a while we have observed the same linear increase of the minimal counts of phase II, as in the experimental data. The numerical solutions obtained for two leukapheresis treatments with approximately one year interval (400 days) between each other is shown in figure 5. The results show a good qualitative agreement with the experimental results [9]. Before the first treatment and between the two treatments $\gamma$ is practically constant (varies approximately from 0.1 to 0.099), maintaining itself in the aperiodic region of the $\gamma$-bifurcation diagram, with no change of attractor (see figure (4). 


\section{Conclusions}

The Mackey-Glass model for the blood-forming process reproduces the main features observed in the peripheral blood-cell counts for normal individuals and pathologies like cyclic leukocytosis and the disorder that characterizes the accumulation of cells observed in a patient diagnosed with leukemia (CML) in phase I and II. We have shown that the onset of leukemia is related to special intermediate values of the time-delay parameter, which seem to correspond to quasi-periodic behaviors. From the bifurcation diagram as a function of the time-delay parameter $\tau$, we observed that the onset of leukemia is associated to the edge of periodic windows in the midst of aperiodic behavior. By varying the death rate parameter we could reproduce the phase II behavior observed in the experimental data, and with a simple cell-removing procedure we simulated the effects of leukapheresis. We have also obtained the bifurcation diagram as a function of the death rate $\gamma$.

\section{Acknowledgements}

We thank A.P. Serbeto, J. Stilck and A. Bernardes for the critical reading of this manuscript. This work was partially supported by the Brazilian Agencies Conselho Nacional para o Desenvolvimento Científico e Tecnológico - CNPq, CAPES and FINEP.

\section{References}

[1] M. Mackey and L. Glass, Science 197, 287-289 (1977).

[2] P.S. Landa and M.G. Rosemblum, Phys.Rev. E 52, R36-39 (1995).

[3] Molecular Biology of The Cell, B. Alberts, D. Bray, J. Lewis, M. Raff, K. Roberts and J. Watson, 2nd. ed., Garland Publishing (1989).

[4] N.S. Cherniak and G.S. Longobardo, New Engl. J. of Medicine 288, 18, 952-957 (1973).

[5] L. Glass and M. C. Mackey, Ann. N.Y. Acad. Sci. 316, 214-35 (1979).

[6] S.I. Rubinow and J.L. Lebowitz, J. Math. Biol. 1, 187-225 (1975).

[7] E.A. King-Smith and A. Morley, Blood 36, 254-262 (1970).

[8] T.E. Wheldon, J. Kirk and H.M. Finlay, Blood 3, 379-387 (1974).

[9] R.A. Gatti, W.A. Robinson, A.S. Deinard, M. Nesbit, J.J. McCullough, M. Ballow and R.A. Good, Blood 41 771-782 (1973). 
[10] N.D. Kazarinoff, P. van den Driessche, Science203, Mar. 30, 1348-49 (1979).

[11] B. Mensour and A. Longtin, Phys. Lett. A 205, 18-24 (1995).

[12] M. Green and R. Savit, Physica D 50, 521-544 (1991).

[13] S. Lepri, G. Giacomelli, A. Politi and F.T. Arecchi, Physica D 70, 235-249 (1993).

[14] M. Le Berre, E. Ressayre, A. Tallet, H.M. Gibbs, D.L. Kaplan and M.H. Rose, Phys. Rev. A 35, 4020-4022 (1987).

[15] S. A. Kauffman, The Origins of Order, Oxford University Press( 1993).

[16] A.A. Morley, A.G. Baikie and D.A.G. Galton, The Lancet 2, 1320-132 (1967).

[17] H. Vodopick, E. M. Rupp, C. L. Edwards, F. A. Goswitz and J.Beauchamp, The New Engl. J. Med 286 284-290 (1972).

[18] B.J. Kennedy, Blood 35, 751-760 (1970).

[19] E.E. Morse, P.P. Carbone, E.J. Freireich, W. Bronson and A. Kliman, Transfusion 6, 175-182 (1966). 


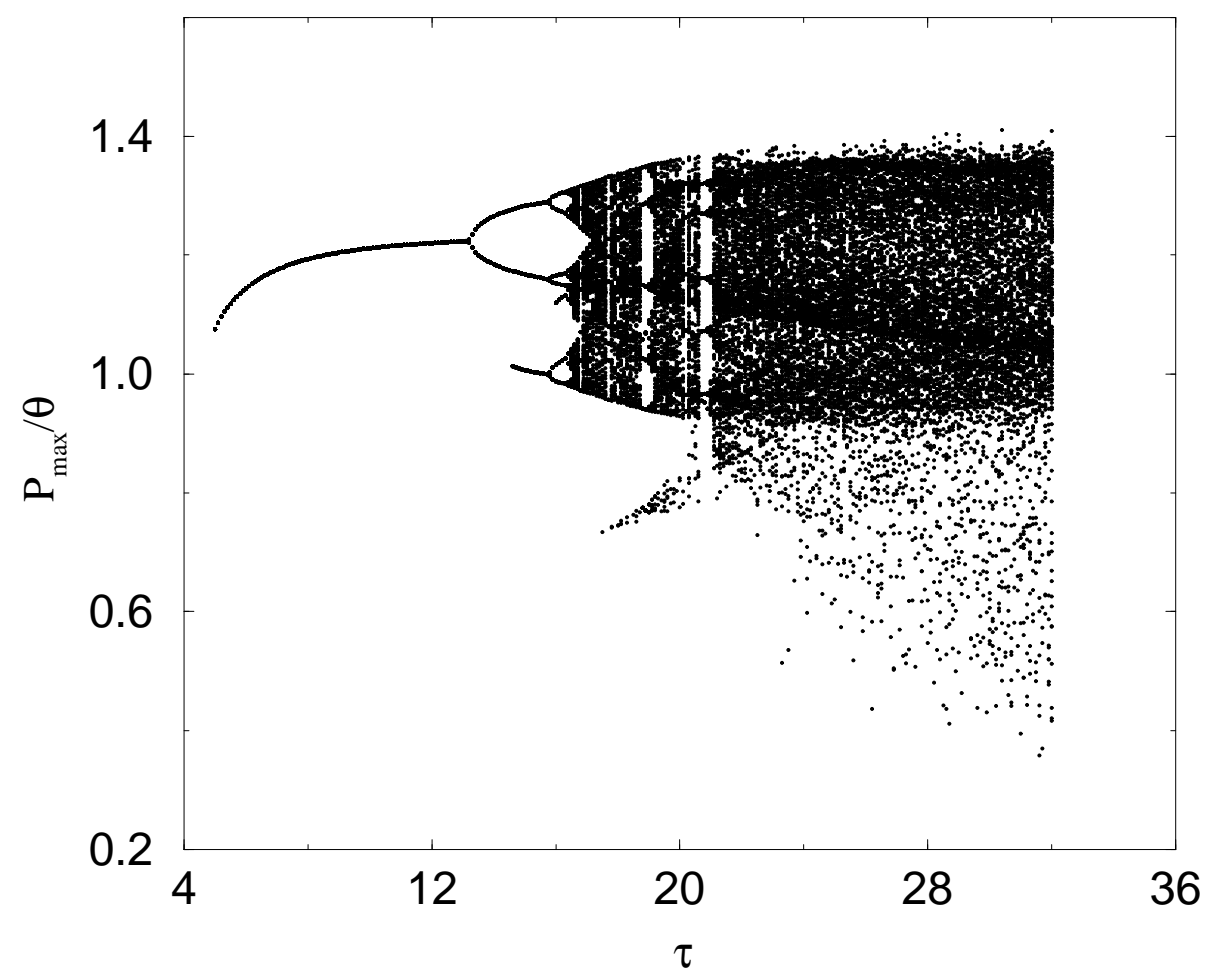

Figure 1: Bifurcation diagram as a function of the time delay parameter $\tau$, for the Mackey-Glass time delayed differential equation using $\beta_{0}=0.2$ per day, $\theta=1.6 \times 10^{-6}, n=10$ and $\gamma=0.1$. 

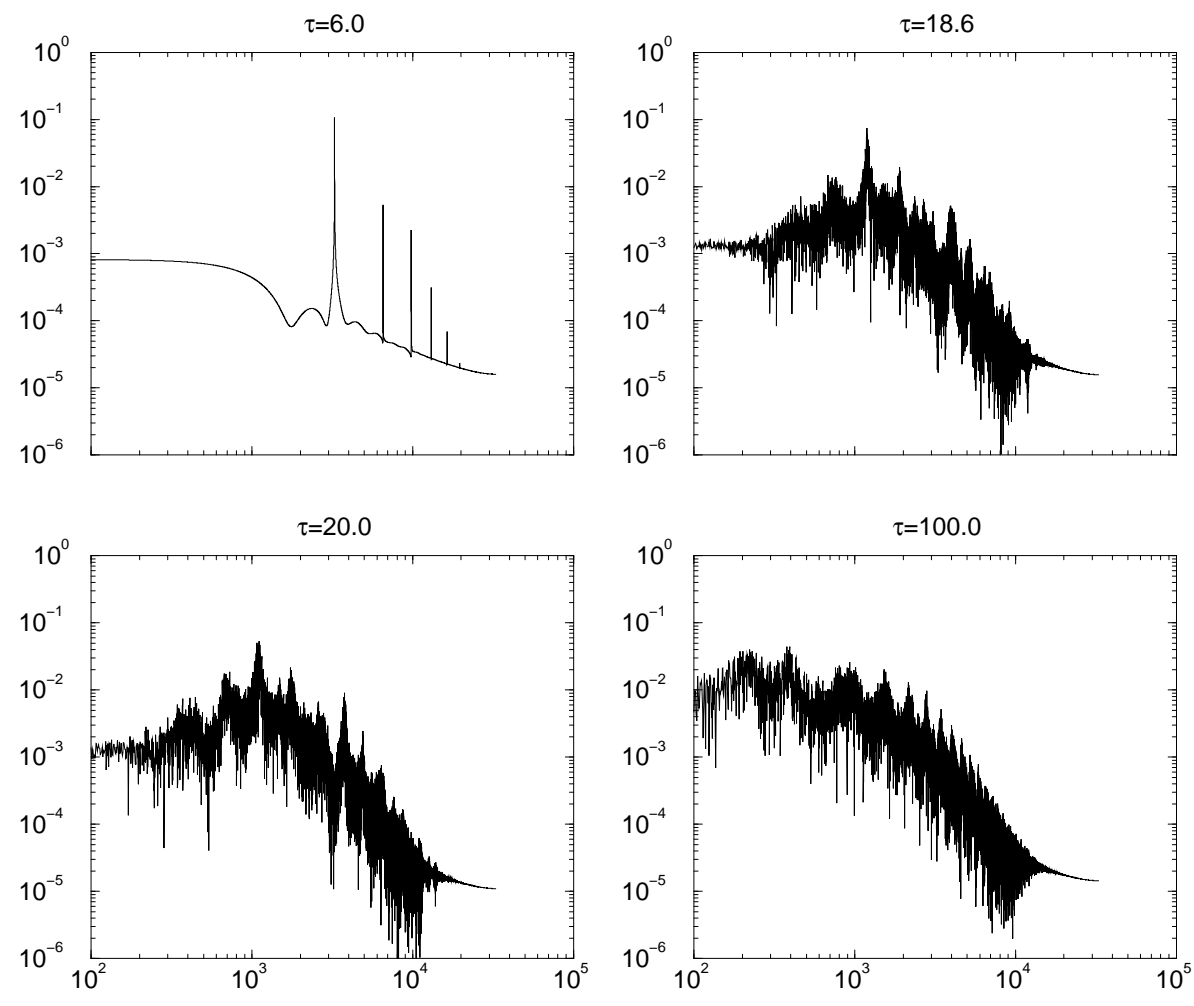

Figure 2: Power spectra of the numerical solutions of the MG equation for periodic $(\tau=6)$, quasi-periodic $(\tau=18.6$ and $\tau=20)$ and chaotic $(\tau=100)$ regimes. 


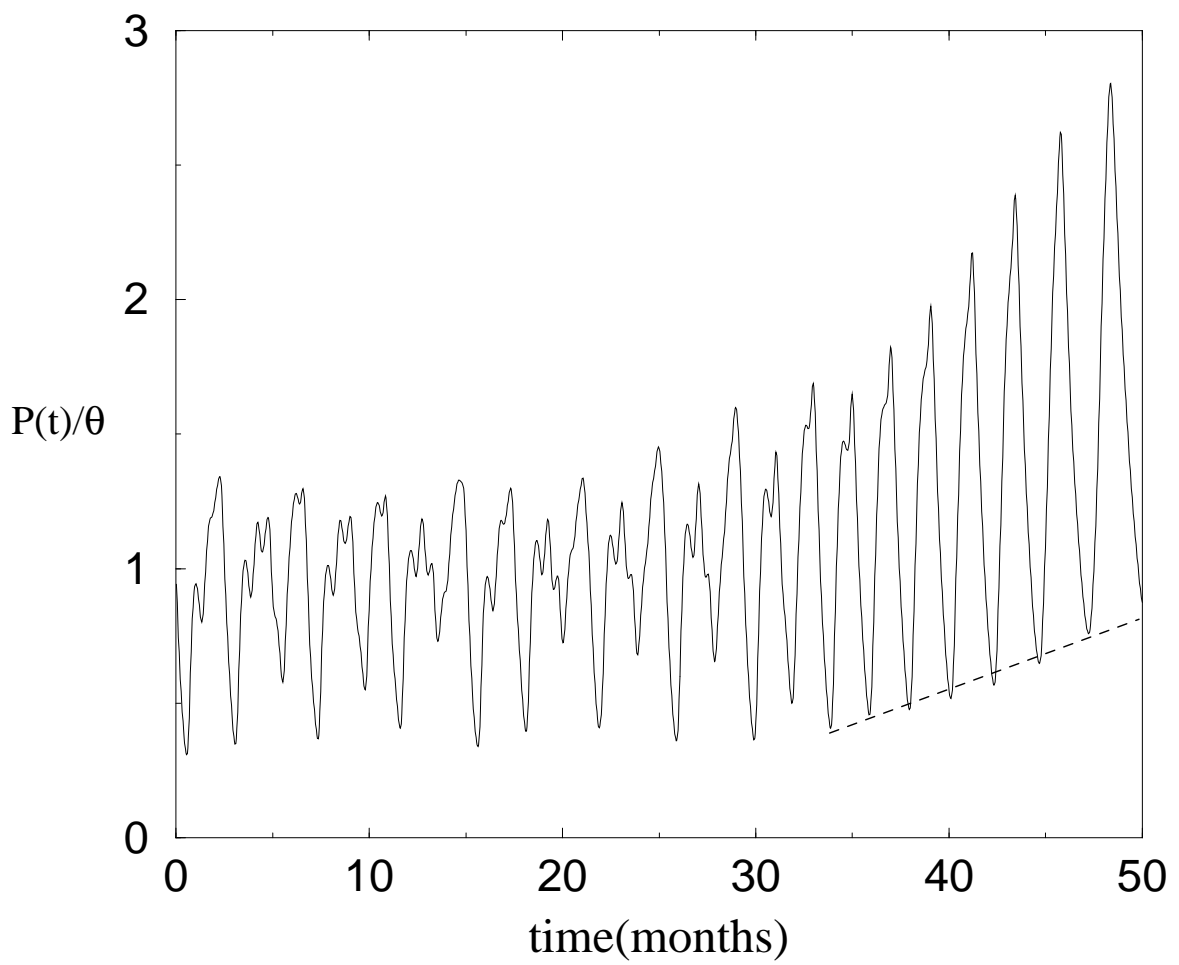

Figure 3: Numerical solution showing qualitative behavior very similar to phases I and II of leukemia [9]. 


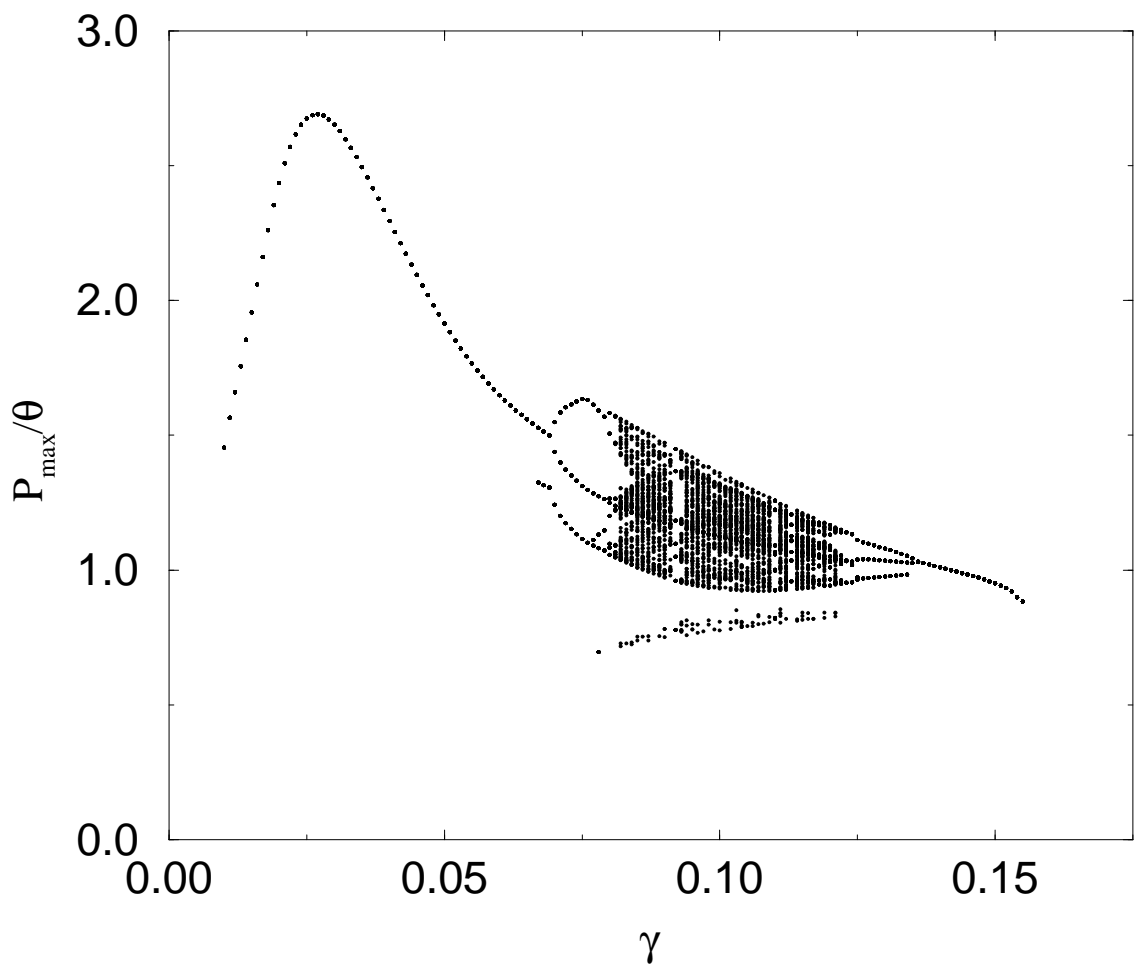

Figure 4: Bifurcation diagram as a function of $\gamma$, obtained for the same set of parameters of figure 1, for $\tau=20$. 


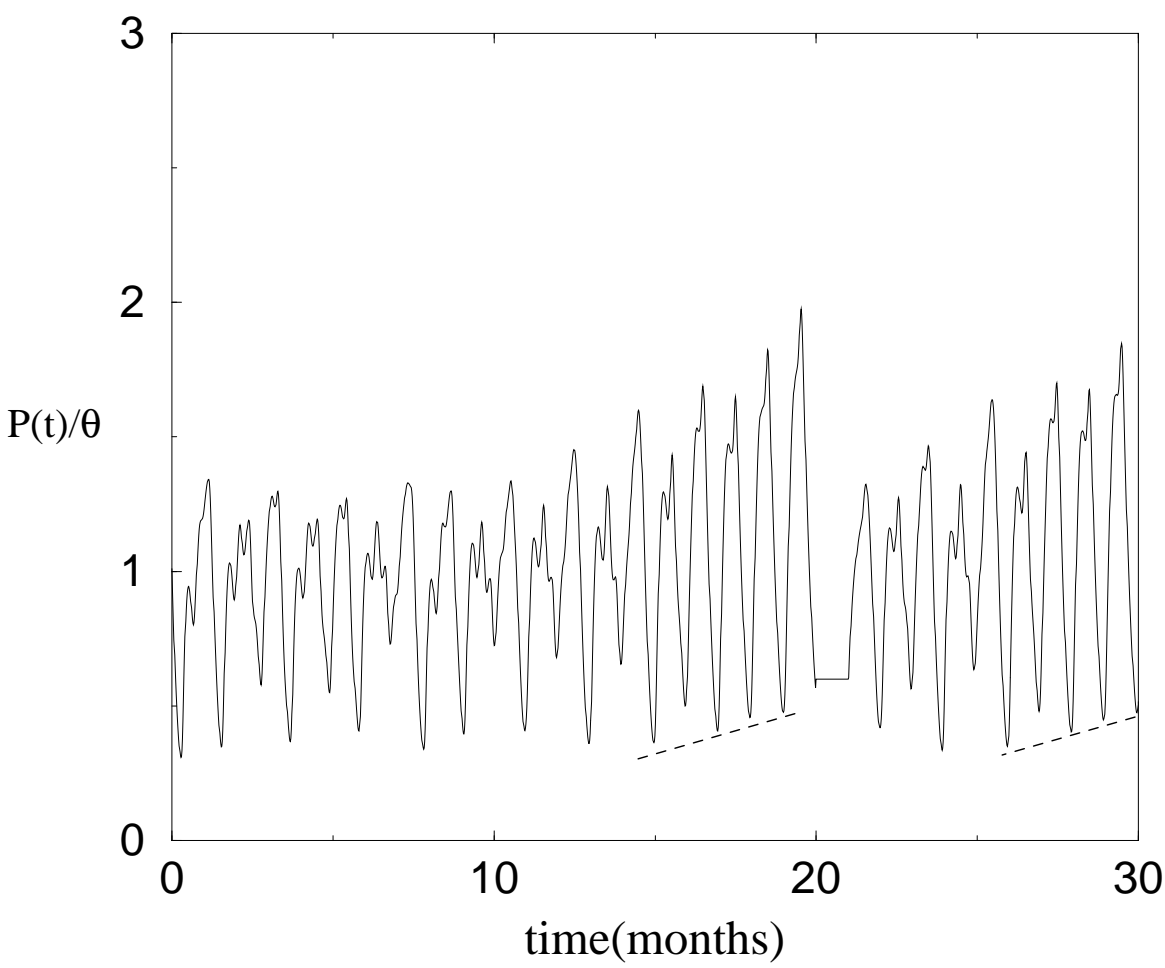

Figure 5: Simulation of two Leukapheresis (I and II) considering the same set of parameters of figure 1, with a time interval of 400 days between the two treatments. Since the disturbance continues to act after the treatment, after some time we observe the same increase of the minima densities, as observed in the experimental data shown in figure 1 of ref. 9 . 